\title{
In vitro susceptibility of Escherichia coli strains isolated from urine samples obtained in mainland China to fosfomycin trometamol and other antibiotics: a 9-year surveillance study (2004-2012)
}

Bei Lai ${ }^{1,4+}$, Bo Zheng ${ }^{2+}$, Yun Li ${ }^{2}$, Sainan Zhu ${ }^{3}$ and Zhaohui Tong ${ }^{1 *}$

\begin{abstract}
Background: As a result of extensive use of fluroquinlones and cephalosporins, urinary tract pathogens producing extended-spectrum beta-lactamase (ESBL) pose a considerable clinical challenge in the treatment of UTIs. In the present study we retrospectively assessed the susceptibility of $E$. coli strains to fosfomycin trometamol and other antibiotics commonly used for the treatment of such infections.

Methods: A total of 908 nonreplicate clinical E. coli urinary isolates were collected from 20 Chinese hospitals over four consecutive 1-year periods between October 2004 and June 2012. Susceptibility to antimicrobial agents fosfomycin trometamol, piperacillin-tazobactam, cefuroxime, cefotaxime, cefepime, imipenem, amikacin, levofloxacin, and nitrofurantoin was determined using the agar dilution method. A reference strain E. coli (ATCC 25922) was used as a positive control. Results were analyzed using Chi-square test or Fisher's exact tests.

Results: Fosfomycin trometamol, piperacillin-tazobactam, amikacin, and imipenem were consistently the most active agents against most of the isolates. There was a decline in susceptibility to cefuroxime, cefotaxime, and cefepime between 2004 and 2010. We showed that 528 of the 908 E. coli isolates (58.1\%) produced ESBLs. The ESBL-positive rates increased from 41.7\% in 2004-2005 to 60.9\% in 2011-2012. ESBL-producing E. coli isolates showed significantly higher resistance rates to levofloxacin than the ESBL-negative isolates. Fosfomycin trometamol, piperacillin-tazobactam, amikacin, and imipenem had good activity against both levofloxacin-susceptible and levofloxacin- nonsusceptible isolates (sensitivity rate $>90 \%$ ). However susceptibility of levofloxacin-resistant isolates to cefuroxime, cefotaxime, cefepime, amikacin, and nitrofurantoin was significantly lower than that of levofloxacin-susceptible isolates.

Conclusions: Owing to the increase in the bacterial resistance across the world, the European Urology Association has recommended fosfomycin trometamol as the drug of choice in its Guidelines on Urological Infections released in 2013. Our results confirm this recommendation for use in China and continued monitoring of the susceptibility of $E$. coli to fosfomycin trometamol is need with the widely use of the drug in China.
\end{abstract}

\section{Background}

Urinary tract infections (UTIs) are among the most common conditions, which require diagnostic and therapeutic intervention. Escherichia coli is to date the most frequent uropathogen, which accounted for $30.7 \%$ and $51.5 \%$ of all UTIs in men and women, respectively, during 2010 in

\footnotetext{
* Correspondence: tongzhh@hotmail.com

${ }^{\dagger}$ Equal contributors

${ }^{1}$ Department of Respiratory and Critical Care Medicine, Beijing Institute of Respiratory Medicine, Beijing Chaoyang Hospital, Capital Medical University, Beijing 100020, PR of China

Full list of author information is available at the end of the article
}

China [1]. Fluoroquinolones and cephalosporins were the most commonly used antibiotics for the treatment of UTIs in China. However, in the recent years, many resistant strains have emerged because of the use of broadspectrum cephalosporins. In particular, strains producing extended-spectrum beta-lactamase (ESBL) pose a considerable clinical challenge in the treatment of UTIs.

Fosfomycin has been extensively used in several European countries for the treatment of uncomplicated UTIs since 1988, but it was not available in China until recently. This study aimed to reassess the susceptibility 
of $E$. coli strains isolated from patients with UTIs between October 2004 and June 2012 to fosfomycin trometamol and other antibiotics commonly used for the treatment of such infections.

\section{Methods}

\section{Bacterial isolates}

A total of 908 nonreplicate clinical E. coli urinary isolates were collected from 20 widely dispersed tertiary Chinese hospitals over four 1-year periods (October 2004-September 2005, January 2007-December 2007, July 2009-June 2010, and July 2011-June 2012) and were sent to the Institute of Clinical Pharmacology, Peking University First Hospital; the isolates were stored at $-80^{\circ} \mathrm{C}$ until further analysis. The isolates were stored at $-80^{\circ} \mathrm{C}$ until further analysis. The strains collected from 20 widely dispersed tertiary Chinese hospitals participating in the Ministry of Health National Antimicrobial Resistance Surveillance Net study. These hospitals are located in 15 different provinces in mainland China. The number of isolates from per hospital per year was from 4 to 26 .

\section{Antimicrobial susceptibility testing}

Susceptibility to antimicrobial agents was determined using the agar dilution method according to the Clinical and Laboratory Standards Institute [2]. The following antimicrobial agents were tested: fosfomycin trometamol, piperacillin-tazobactam, cefuroxime, cefotaxime, cefepime, imipenem, amikacin, levofloxacin, and nitrofurantoin. ESBL)-producing isolates were detected according to previously described methods [2]. The reference strain E. coli ATCC 25922 was used as the positive control.

\section{Statistical analyses}

Statistical tests were performed using Social Sciences software for Windows Version 14.0 (SPSS, Inc., Chicago, IL, USA). Enumeration data were expressed as percentage values. The differences in susceptibility between the groups were compared using the Chi-square test or Fisher's exact test. The differences between the groups were considered significant if the p-values were smaller than 0.05(two-sided test). The Bonferroni method was used to adjust the significant levels $(0.05 / 6=0.0083)$ in multiple comparisons between any two levels of the susceptibility outcome.

\section{Results}

\section{Comparison of antimicrobial susceptibilities between 2004 and 2012}

Fosfomycin trometamol, piperacillin-tazobactam, amikacin, and imipenem were consistently the most active agents against most of the isolates. We observed a decline in the susceptibility to cefuroxime, cefotaxime, and cefepime from 2004 to 2010. In particular, the susceptibility to cefepime decreased significantly from $88.1 \%$ during October 2004-September 2005 to $61.5 \%$ during July 2009-June 2010 ( $\mathrm{p}<0.0001)$. Over the same period, the minimum inhibitory concentration inhibiting $90 \%$ of the strains $\left(\mathrm{MIC}_{90}\right)$ increased from $16 \mathrm{mg} / \mathrm{L}$ to $64 \mathrm{mg} / \mathrm{L}$. Susceptibility to nitrofurantoin was $78.6 \%$ during October 2004-September 2005, which increased to $83.0 \%$ during January 2007-December 2007 and to $91.6 \%$ during July 2009-June 2010 period ( $<<0.0001)$ (Table 1).

\section{Comparison of antimicrobial susceptibilities to}

ESBL-producing strains of $E$. coli

In this study, 528 E. coli isolates (58.1\%) produced ESBLs. The ESBL-positive rates were $41.7 \%$ (70/168), $58.7 \%$ (159/271), 66.0\% (173/262), and 60.9\% (126/207) in the four

Table 1 Comparison of antimicrobial susceptibilities between 2004 and 2012

\begin{tabular}{|c|c|c|c|c|c|c|c|c|c|}
\hline \multirow{2}{*}{ Antibiotic $^{a}$} & \multicolumn{2}{|c|}{$10 / 2004-9 / 2005(n=168)$} & \multicolumn{2}{|c|}{$1 / 2007-12 / 2007(n=271)$} & \multicolumn{2}{|c|}{$7 / 2009-6 / 2010(n=262)$} & \multicolumn{2}{|c|}{$7 / 2011-6 / 2012(n=207)$} & \multirow{2}{*}{$\mathbf{p}$} \\
\hline & S (\%) & $\mathrm{MIC}_{90}(\mathrm{mg} / \mathrm{L})$ & S (\%) & $\mathrm{MIC}_{90}(\mathrm{mg} / \mathrm{L})$ & S (\%) & $\mathrm{MIC}_{90}(\mathrm{mg} / \mathrm{L})$ & $\mathrm{S}(\%)$ & $\mathrm{MIC}_{90}(\mathrm{mg} / \mathrm{L})$ & \\
\hline FOS & 99.4 & 2 & 95.9 & 1 & 95.0 & 4 & $93.2^{*}$ & 8 & 0.030 \\
\hline TZP & 94.0 & 16 & 97.8 & 8 & 92.7 & 16 & 94.7 & 8 & 0.058 \\
\hline CXM & 42.3 & 512 & 32.8 & 512 & $27.5^{*}$ & 512 & 30.9 & 512 & 0.015 \\
\hline CTX & 53.0 & 128 & $37.6^{*}$ & 256 & $30.9^{*}$ & 512 & $36.2^{*}$ & 512 & $<0.001$ \\
\hline FEP & 88.1 & 16 & $76.4^{*}$ & 32 & $61.5^{* * * *}$ & 64 & $75.4^{* * * * *}$ & 32 & $<0.001$ \\
\hline IMP & 100 & 0.25 & 99.6 & 0.25 & 99.6 & 0.125 & 99.5 & 0.125 & 1.000 \\
\hline AMK & 94.6 & 4 & 95.6 & 4 & 92.7 & 8 & 93.2 & 4 & 0.519 \\
\hline NIT & 78.6 & 64 & 83.0 & 64 & $91.6^{*, * * *}$ & 32 & $88.9^{*}$ & 64 & $<0.001$ \\
\hline LVX & 31.0 & 32 & 28.0 & 32 & 28.2 & 32 & 32.4 & 32 & 0.693 \\
\hline
\end{tabular}

${ }^{a}$ Drug abbreviations: FOS, fosfomycin trometamol; TZP, piperacillin-tazobactam; CXM, cefuroxime; CTX, cefotaxime; FEP, cefepime; IMP, imipenem; AMK, amikacin; NIT, nitrofurantoin; LVX, levofloxacin.

*compared to $10 / 2004-9 / 2005, \mathrm{p}<0.0083$.

** compared to $1 / 2007-12 / 2007, \mathrm{p}<0.0083$.

*** compared to $7 / 2009-6 / 2010, p<0.0083$. 
periods from 2004 to 2012, respectively. ESBL-producing $E$. coli isolates showed significantly higher resistance rates to cefepime and levofloxacin than the ESBL-negative isolates. Fosfomycin trometamol, piperacillin-tazobactam, imipenem, amikacin, and nitrofurantoin showed good activity against both ESBL-producing and ESB-negative isolates; the sensitivity rates were greater than $85 \%$ (Table 2).

\section{Comparison of susceptibility of levofloxacin-resistant} and levofloxacin-susceptible strains of $E$. coli to various antimicrobial agents

Fosfomycin trometamol, piperacillin-tazobactam, amikacin, and imipenem had good activity against both levofloxacinsusceptible and levofloxacin- nonsusceptible isolates (sensitivity rate $>90 \%$ ). The rate of susceptibility to cefuroxime, cefotaxime, cefepime, amikacin, and nitrofurantoin was significantly lower in the levofloxacin-resistant isolates than in the levofloxacin-susceptible isolates $(\mathrm{p}<0.001)$ (Table 3$)$.

\section{Discussion and conclusion}

E. coli plays an important role in UTI; UTI is one of the most frequently encountered infectious diseases. Cephalosporins and levofloxacin are the widely used agents for the treatment of UTI. The production of ESBLs by $E$. coli is associated with the reduced susceptibility to fluoroquinolones and other antimicrobial agents. The resistance rates of ESBL-producing $E$. coli to levofloxacin in this study were higher than those reported in other studies performed in different countries $[3,4]$. Nitrofurantoin were seldom used in China, it is maybe the reason that the susceptibility of nitrofurantoin increased over time.

Our data confirm that E. coli isolated from Chinese UTI patients remain exclusively susceptible to fosfomycin trometamol. In addition, the ESBL-producing and levofloxacin-resistant strains of $E$. coli were susceptible to fosfomycin trometamol. Our results are similar to

Table 2 Comparison of the susceptibilities of ESBL-producing Escherichia coli strains to various antimicrobial agents

\begin{tabular}{|c|c|c|c|c|c|}
\hline \multirow[t]{2}{*}{ Antibiotic } & \multicolumn{2}{|c|}{$\begin{array}{l}\text { ESBL-positive } \\
\quad(n=528)\end{array}$} & \multicolumn{2}{|c|}{$\begin{array}{l}\text { ESBL-negative } \\
\quad(n=380)\end{array}$} & \multirow{2}{*}{$p$} \\
\hline & S (\%) & $\mathrm{MIC}_{90}(\mathrm{mg} / \mathrm{L})$ & $\mathrm{S}(\%)$ & $\mathrm{MIC}_{90}(\mathrm{mg} / \mathrm{L})$ & \\
\hline FOS & 93.8 & 2 & 98.4 & 1 & 0.001 \\
\hline $\mathrm{TZP}$ & 95.3 & 16 & 95.8 & 8 & 0.707 \\
\hline IMP & 100 & 0.25 & 99.2 & 0.25 & 0.073 \\
\hline AMK & 92.8 & 8 & 95.8 & 4 & 0.061 \\
\hline NIT & 86.2 & 64 & 85.8 & 64 & 0.869 \\
\hline LVX & 17.2 & 32 & 46.8 & 32 & $<0.001$ \\
\hline
\end{tabular}

a Drug abbreviations: FOS, fosfomycin trometamol; TZP, piperacillin-tazobactam; IMP, imipenem; AMK, amikacin; NIT, nitrofurantoin; LVX, levofloxacin; ESBL, extended-spectrum beta-lactamase.
Table 3 Comparison of susceptibility of levofloxacinsusceptible and levofloxacin- nonsusceptible strains of Escherichia coli to various antimicrobial agents

\begin{tabular}{|c|c|c|c|c|c|}
\hline \multirow{2}{*}{ Antibiotic $^{a}$} & \multicolumn{2}{|c|}{ LVX-S $(n=269)$} & \multicolumn{2}{|c|}{ LVX-NS $(n=639)$} & \multirow{2}{*}{$p$} \\
\hline & S (\%) & $\mathrm{MIC}_{90}(\mathrm{mg} / \mathrm{L})$ & S (\%) & $\mathrm{MIC}_{90}(\mathrm{mg} / \mathrm{L})$ & \\
\hline FOS & 98.5 & 0.5 & 94.5 & 4 & 0.007 \\
\hline $\mathrm{TZP}$ & 95.9 & 4 & 95.3 & 8 & 0.688 \\
\hline CXM & 63.2 & 512 & 19.7 & 512 & $<0.001$ \\
\hline CTX & 64.7 & 128 & 27.1 & 512 & $<0.001$ \\
\hline FEP & 91.4 & 8 & 66.7 & 64 & $<0.001$ \\
\hline IMP & 100 & 0.25 & 99.5 & 0.25 & 0.559 \\
\hline AMK & 98.9 & 2 & 92.0 & 8 & $<0.001$ \\
\hline NIT & 96.3 & 16 & 81.7 & 64 & $<0.001$ \\
\hline
\end{tabular}

LVX-S, levofloxacin-susceptible; LVX-NS, levofloxacin-resistant or intermediately. aDrug abbreviations: FOS, fosfomycin trometamol; TZP, piperacillin-tazobactam; CXM, cefuroxime; CTX, cefotaxime; FEP, cefepime; IMP, imipenem; AMK, amikacin; NIT, nitrofurantoin; LVX, levofloxacin.

those reported in recent studies on the same pathogens isolated from other areas of the world [5,6].

Fosfomycin trometamol was excreted renally within 48 hours and urinary titres showed that concentrations $>128 \mathrm{mg} / \mathrm{L}$, sufficient to inhibit most urinary pathogens, were maintained for 24 to 48 hours after a single oral dose ( $\cong 3 \mathrm{~g}$ fosfomycin), but the mean peak plasma concentrations were only 22 to $32 \mathrm{mg} / \mathrm{L}$ [7] The European Urology Association has recommended fosfomycin trometamol, nitrofurantoin and pivmecillinam as the drug of choice in its Guidelines on Urological Infections released in 2013 [8]. An uncontrolled and multicentre study showed clinical efficacy rates for acute uncomplicated cystitis, recurrent lower urinary tract infection and complicated lower urinary tract infection were 94.71, 77.22\% and 62.69\% [9]. Continued monitoring of the susceptibility of E.coli to fosfomycin trometamol is needed with the widely use of the drug in China.

Competing interests

The authors declare that they have no conflict of interest.

Authors' contributions

$B L, B Z$ and $Y L$ carried out the antimicrobial susceptibility testing and drafted the manuscript. SZ participated in the design of the study and performed the statistical analysis. ZT conceived of the study, and participated in its design and coordination and helped to draft the manuscript. All authors read and approved the final manuscript.

\section{Author details}

'Department of Respiratory and Critical Care Medicine, Beijing Institute of Respiratory Medicine, Beijing Chaoyang Hospital, Capital Medical University, Beijing 100020, PR of China. Institute of Clinical Pharmacology, Peking University First Hospital, Beijing 100034, PR of China. ${ }^{3}$ Department of Biostatistics, Peking University First Hospital, Beijing 100034, PR of China. ${ }^{4}$ Department of Rheumatology and Immunology, Beijing Hosptial, Beijing 100730, PR of China. 
Received: 30 September 2013 Accepted: 4 February 2014

Published: 6 February 2014

\section{References}

1. Zheng B, LV Y: Ministry of health national antimicrobial investigation Net 2010 report: bacterial distribution and resistance in urinary tract infections in male. Chin J Clin Pharmacol 2011, 27(12):905-912.

2. Clinical and Laboratory Standards Institute: Performance standards for antimicrobial susceptibility testing; M100-S22. Wayne, PA: Clinical and Laboratory Standards Institute; 2012.

3. Muratani T, Matsumoto T: Bacterial resistance to antimicrobials in urinary isolates. Int J Antimicrob Agents 2004, 24(Suppl 1):S28-S31.

4. Ko KS, Suh JY, Peck KR, Lee MY, Oh WS, Kwon KT, Jung DS, Lee NY, Song JH: In vitro activity of fosfomycin against ciprofloxacin-resistant or extended-spectrum beta-lactamase-producing Escherichia coli isolated from urine and blood. Diagn Microbiol Infect Dis 2007, 58(1):111-115.

5. Lu PL, Liu YC, Toh HS, Lee YL, Liu YM, Ho CM, Huang CC, Liu CE, Ko WC, Wang $\mathrm{JH}$, et al: Epidemiology and antimicrobial susceptibility profiles of gram-negative bacteria causing urinary tract infections in the Asia-Pacific region: 2009-2010 results from the study for monitoring antimicrobial resistance trends (SMART). Int J Antimicrob Agents 2012, 40(Suppl):S37-S43.

6. Liu HY, Lin HC, Lin YC, Yu SH, Wu WH, Lee YJ: Antimicrobial susceptibilities of urinary extended-spectrum beta-lactamase-producing Escherichia coli and Klebsiella pneumoniae to fosfomycin and nitrofurantoin in a teaching hospital in Taiwan. J Microbiol Immunol Infect 2011, 44(5):364-368.

7. Patel SS, Balfour JA, Bryson HM: Fosfomycin tromethamine. A review of its antibacterial activity, pharmacokinetic properties and therapeutic efficacy as a single-dose oral treatment for acute uncomplicated lower urinary tract infections. Drugs 1997, 53(4):637-656.

8. European Association of Urology, Guidelines on Urological Infections. [http://www.uroweb.org/fileadmin/guidelines/Total_file_2013_large_ guidelines_prints.pdf].

9. Qiao LD, Zheng B, Chen S, Yang Y, Zhang K, Guo HF, Yang B, Niu YJ, Wang $Y$, Shi BK, et al: Evaluation of three-dose fosfomycin tromethamine in the treatment of patients with urinary tract infections: an uncontrolled, open-label, multicentre study. BMJ Open 2013, 3(12):e004157.

doi:10.1186/1471-2334-14-66

Cite this article as: Lai et al.: In vitro susceptibility of Escherichia coli strains isolated from urine samples obtained in mainland China to fosfomycin trometamol and other antibiotics: a 9-year surveillance study (2004-2012). BMC Infectious Diseases 2014 14:66.

\section{Submit your next manuscript to BioMed Central and take full advantage of:}

- Convenient online submission

- Thorough peer review

- No space constraints or color figure charges

- Immediate publication on acceptance

- Inclusion in PubMed, CAS, Scopus and Google Scholar

- Research which is freely available for redistribution 